\title{
Assessment of myocardial inflammation post- infarct with PET/MRI: Getting into the nitty-gritty
}

\author{
Daniel Juneau, MD, ${ }^{\mathrm{a}, \mathrm{b}}$ and Matthieu Pelletier-Galarneau, MD, MSc, ${ }^{\mathrm{c}, \mathrm{d}}$ \\ a Department of Medical Imaging, Centre Hospitalier de l'Université de Montréal, Montreal, \\ Quebec, Canada \\ b University of Ottawa Heart Institute, Ottawa, Canada \\ c Department of Medical Imaging, Institut de Cardiologie de Montréal, Montreal, Canada \\ d Gordon Center for Medical Imaging, Massachusetts General Hospital, Harvard Medical School, \\ Boston, MA
}

Received Jan 22, 2021; accepted Jan 22, 2021

doi: $10.1007 / \mathrm{s} 12350-021-02558-1$

See related articles, pp. 1304-1314 and
pp. 1315-1325

Integrated hybrid positron emission tomographymagnetic resonance imaging (PET/MRI) systems were first introduced a little over 10 years ago. Widespread adoption and utilization of PET/MRI has been hampered by several factors, including the substantial cost of the systems, challenges in obtaining accurate attenuation maps, and the overall effectiveness of PET-computed tomography (PET/CT) systems. To date, few PET/MRI applications stand out, most of which are driven by the overpowering superiority of MRI over CT in specific contexts. Neurology, neuro-oncology, prostate cancer, gynecologic malignancies, and pediatric imaging are good examples of areas where PET/MRI can shine. ${ }^{1}$ Cardiovascular imaging is another example where PET/ MRI systems could find valuable applications. After all, PET and MRI often play complementary roles in several cardiovascular pathologies, such as cardiac inflammation, vasculitis, cardiac tumors, and viability assessment. ${ }^{2-4}$ In addition, with PET/MRI systems, it is possible to apply corrections accounting for both

Reprint requests: Daniel Juneau, MD, Department of Medical Imaging, Centre Hospitalier de l'Université de Montréal, 1000 rue SaintDenis, Montreal, Quebec H2X 0C1, Canada; daniel.juneau@umontreal.ca

J Nucl Cardiol 2022;29:1326-8.

$1071-3581 / \$ 34.00$

Copyright (c) 2021 American Society of Nuclear Cardiology. breathing and cardiac motion, ${ }^{5}$ allowing for improved spatial resolution and quantification accuracy.

One of the cardiovascular applications that has been evoked is imaging of post-ischemic inflammation in the heart. Acute myocardial infarction (MI) is associated with an inflammatory response, essential to clear dead cells and activate reparative pathways. ${ }^{6}$ However, excessive inflammatory response may be associated with suboptimal healing and undesirable left ventricular remodeling. ${ }^{6,7}$ Wollenweber et al have previously demonstrated the feasibility of imaging the inflammatory tissue response in acute MI in humans using multimodality imaging. ${ }^{8}$ In their study, 15 patients underwent both cardiac MRI and PET/CT imaging with fluorodeoxyglucose (FDG) within 7 days following MI and following a typical myocardial suppression protocol which included prolonged fasting ( $>12$ hours) and intravenous heparin injection. On MRI, they observed edema extending beyond the area of late gadolinium enhancement (LGE). They also showed increased glucose metabolic rates in the infarcted segments (LGE) compared to normal segments and edema segments. Most of the published literature up to now has concentrated on the infarct and peri-infarct areas, yet it is known that an inflammatory response is also present in the remote myocardium. ${ }^{9}$ Despite the central role inflammation plays in post-MI healing, very few studies have investigated the role of PET/MRI in that setting. Thus, our understanding of this phenomenon and its potential application as a target for therapeutic intervention is currently limited.

In this issue of the Journal, Smailovic et al and Wilk et al present companion articles which aim to improve our understanding of myocardial inflammation post infarction through the use of hybrid PET/MRI 
imaging. In the first article, entitled "Simultaneous measurements of myocardial glucose metabolism and extra-cellular volumes with hybrid PET/MRI using concurrent injections of Gd-DTPA and FDG', the authors investigate three important technical questions related to PET/MRI imaging of myocardial inflammation: (1) How does the gadolinium-based contrast agent (GBCA) injection protocol impact extra-cellular volume (ECV) quantification with MRI? (2) Does the use of a physiological myocardial FDG suppression protocol affect ECV quantification? (3) Does the simultaneous injection of FDG and a GBCA impact the chemical stability of the compounds? To investigate these questions, they performed in vivo PET/MRI imaging in five healthy canines using three different GBCA protocols: (1) the standard bolus injection most commonly used in human patients, (2) a constant 60 minutes infusion with no bolus, and (3) a bolus followed by a 30 minutes constant infusion. Suppression of physiological myocardial FDG uptake was achieved through a continuous infusion of IV lipid and an injection of IV heparin, and all three GBCA injection protocols were tested with and without the suppression protocol, for a total of six acquisitions per subject.

The authors reported that, in healthy myocardium, there are no significant differences between the three GBCA injection protocols, except in the first 15 minutes following bolus injection, when ECV may be underestimated. This is likely related to the fact that distribution of GBCA is not at equilibrium during that period, a requirement for accurate ECV quantification. Unsurprisingly, they also reported that a concomitant injection of FDG and GBCA did not alter the stability of FDG, based on the testing they performed. When it comes to the potential impact of the glucose suppression protocol, however, the results are a bit more obscure. When the authors analyzed the impact of the suppression protocol in each individual group (divided by GBCA injection protocols), they reported no statistically significant effect. However, when pooling all GBCA protocols, they observed significantly higher ECV with suppression protocol compared to measurements obtained without suppression protocol $(0.21 \pm 0.02$ vs $0.18 \pm$ $0.01, p=.002)$. Although the difference is statistically significant, it is relatively small and possibly not clinically significant. Nonetheless, this result raises an important question as most cardiovascular applications of PET/MRI imaging are likely to be performed with suppression protocol. Unfortunately, the sample size is too small to determine whether a specific GBCA injection protocol may be less affected. The authors postulated that this finding may be partially accounted for by the change in osmotic pressure secondary to the heparin injection. Further studies are needed to elucidate this question. It must be pointed out that the myocardial suppression protocol used in the study, specifically the use of a lipid infusion, is not the standard of care in humans. Thus, whether the current standard of care for myocardial suppression in humans, usually consisting of prolonged fasting, high-fat-low-carbohydrate diet, and heparin injection, significantly affects ECV quantifications remains unknown.

In the companion paper, entitled "Tracking the progress of inflammation with PET/MRI in a canine model of myocardial infarction', the same group, building on the technical knowledge gained in the first paper, set out to investigate the use of hybrid PET/MRI to characterize changes in the infarcted and remote myocardium following acute MI. Eight canines underwent serial PET/MRI imaging following permanent left anterior descending coronary snare ligature. Imaging was performed at baseline and at 5 additional time points over a 40-day period. The authors used a constant infusion of FDG and GBCA over 60 minutes and suppressed physiological myocardial glucose uptake using the previously described protocol. They then looked at ECV and $K_{\mathrm{i}}$ (the influx constant of FDG, a surrogate of glucose metabolism) in the infarcted and remote myocardium. The author further divided the infarcted tissue in infracted obstructed tissue (IOT), the area of infarct where microvascular obstruction is observed and tracer delivery likely compromised, vs the infarcted not obstructed tissue (INOT).

As expected, ECV was significantly elevated in the INOT at all time points when compared to the baseline. $K_{\mathrm{i}}$ was also significantly increased in the days following MI when compared to baseline. Interestingly, while ECV remained elevated at all time points, $K_{\mathrm{i}}$ values peaked at the 3 days time point, after which they started to decrease with a return to baseline values after approximately 40 days. This discrepancy may potentially indicate that $K_{\mathrm{i}}$ changes are a better reflection of the transient inflammatory response to an acute injury, while ECV changes might better reflect more chronic ischemic changes. In remote myocardium, there was an increase in ECV compared to baseline at 14- and 21 days post-MI, which then appeared to normalize by day 40. Although a slight trend was observed toward a mild increase in $K_{\mathrm{i}}$ in the remote myocardium at 14- and 21 days post-MI, this failed to reach statistical significance. Only a few data points were available for IOT, making it difficult to draw any meaningful conclusions. Interestingly, the data demonstrated that the ECV in the INOT reached equilibrium at 50 minutes for most time points, validating that a constant 60 minutes infusion was likely sufficient to obtain good wash-in. However, based on the limited data available, it does appear that equilibrium in 
the IOT had not been reached at 60 minutes, and ECV values thus obtained are unlikely to be accurate.

Overall, the findings presented are mostly consistent with previously published studies. We congratulate the authors for their study designs, which included paired comparison of several protocols and longitudinal assessments. However, many questions remain unanswered and it would be highly premature to draw any hard conclusions from the presented data. This work should be seen as a proof of concept that such a PET/ MRI study is possible in vivo and opens the door to similar studies in human subjects. It demonstrates the unique potential of hybrid PET/MRI as a tool to help us better understand post-MI changes in the infarct and the remote myocardium, if some technical hurdles can be overcome. The data presented provides good evidence that a bolus injection, either of GBCA or FDG, is unlikely to be enough to obtain accurate data in areas of severely compromised perfusion (IOT), and that even in less severely impacted areas of infarct (INOT), a prolonged infusion is required to reach equilibrium. This should act as a warning and help the community avoid this pitfall when designing future studies. At the same time, this work highlights the potential confounding effects of combining both modalities, and underscores the need for further research, both of a clinical and technical nature. For example, the impact of the glucose suppression protocol on ECV will need to be better understood. What is its mechanism, and is it present when using a "traditional" human suppression protocol? This is likely to be only one of many questions that will need to be answered. Lastly, the inflammatory changes observed in the remote myocardium provide another proof that a pathological process is at work in this tissue in the days and weeks following MI. While this has been shown in this study and others, ${ }^{10}$ it is still poorly understood how this impacts the healing process and prognosis. A better understanding of this phenomenon might provide us with new targets for treatment or help us optimize current ones. While it is too early to draw any clinically meaningful conclusion at this point, PET/MRI does appear to be ideally positioned to help us better understand the pathophysiology of post-infarct remodeling if we can unlock its full potential.

\section{Disclosures}

D.J. has received consultant fees from AbbVie, Advanced Accelerator Applications and Pfizer. M.P.-G. is supported by a Junior 1 Research Award from the Fonds de Recherche du Québec-Santé(FRQS). M.P.-G. has received consultant fees from Pfizer.

\section{References}

1. Hope TA, Fayad ZA, Fowler KJ et al (2019) State of the art PET/ MRI: Applications and limitations-Summary of the First ISMRM/SNMMI Co-Provided Workshop on PET/MRI. J Nucl Med. https://doi.org/10.2967/jnumed.119.227231

2. Pelletier-Galarneau M, Ruddy TD (2019) PET/CT for diagnosis and management of large-vessel vasculitis. Curr Cardiol Rep 21:34. https://doi.org/10.1007/s11886-019-1122-z

3. Martineau P, Pelletier-Galarneau M, Juneau D et al (2018) Molecular imaging of cardiac sarcoidosis. Curr Cardiovasc Imaging Rep 11:6-17

4. Rischpler C, Siebermair J, Kessler L et al (2020) Cardiac PET/ MRI: Current clinical status and future perspectives. Semin Nucl Med 50:260-9. https://doi.org/10.1053/j.semnuclmed.2020.02.012

5. Munoz C, Neji R, Cruz G et al (2018) Motion-corrected simultaneous cardiac positron emission tomography and coronary MR angiography with high acquisition efficiency. Magn Reson Med 79:339-50. https://doi.org/10.1002/mrm.26690

6. Frangogiannis NG (2012) Regulation of the inflammatory response in cardiac repair. Circ Res 110:159-73. https://doi.org/10. 1161/CIRCRESAHA. 111.243162

7. van der Laan AM, Nahrendorf M, Piek JJ (2012) Healing and adverse remodelling after acute myocardial infarction: Role of the cellular immune response. Heart Br Card Soc 98:1384-90. https://d oi.org/10.1136/heartjnl-2012-301623

8. Wollenweber T, Roentgen P, Schäfer A et al (2014) Characterizing the inflammatory tissue response to acute myocardial infarction by clinical multimodality noninvasive imaging. Circ Cardiovasc Imaging 7:811-8. https://doi.org/10.1161/CIRCIMA GING.114.001689

9. Lee WW, Marinelli B, van der Laan AM et al (2012) PET/MRI of inflammation in myocardial infarction. J Am Coll Cardiol 59:15363. https://doi.org/10.1016/j.jacc.2011.08.066

10. Chan W, Duffy SJ, White DA et al (2012) Acute left ventricular remodeling following myocardial infarction. JACC Cardiovase Imaging 5:884-93. https://doi.org/10.1016/j.jcmg.2012.03.015

Publisher's Note Springer Nature remains neutral with regard to jurisdictional claims in published maps and institutional affiliations. 